\title{
Microwave Synthesis of Silver Nano Particles
}

\author{
Sonali Saha $^{a *}$, M.M. Malik ${ }^{b}$ M.S. Qureshic \\ Department of Physics, Maulana Azad National Institute of Technology (MANIT) \\ Bhopal, India.

\begin{abstract}
*Corresponding author Email address: ${ }^{*}$ pronit01@gmail.com,
bmanzar.malik@gmail.com, ${ }^{\mathrm{c}}$ shumsqureshi@rediffmail.com
\end{abstract}

Keywords: Green synthesis, Silver nanoparticle, Ocimum leaf extract, Rapid synthesis using Microwave.

\begin{abstract}
In this work, a green route for synthesis of $\mathrm{Ag}$ nanoparticles is presensted. For the synthesis of Ag nanoparticles, tulasi leaf extract (Ocimum leaf) in combination with microwave irradiation was used which yielded stable spherical Ag nanoparticle in the range of 5-50 nm. Surface morphology of nanoparticle was analyzed by XRD and TEM. UV-Vis analysis was also carried out to characterize the synthesized nanoparticles. The main feature of the process is that it is carried out in a very short span of time in comparison to other conventional physical, chemical and biological methods. Microwave assisted synthesis suppresses the enzymatic action to keep the process easy, fast and eco-friendly.
\end{abstract}

\section{Introduction}

In modern material science the field of nanotechnology is one of the most active areas of research. Based on new and improved specific characteristics such as size, distribution and morphology, the nanoparticles exhibit completely improved properties. The applications of nanoparticles and nanomaterials are being explored widely [1-3]. Nanocrystalline silver particles have found tremendous applications in the field of high sensitivity bio molecular detection and diagnostics [4], 
antimicrobials and therapeutics [5-6], catalysis [7] and micro-electronics [8]. Silver nanoparticles are already been used in antibacterial clothing, burn ointments and coating for medical devices, because of their mutation-resistant antimicrobial activity [9]. Although there is a need for economic, commercially viable as well as environmentally clean approach for synthesis of silver nanoparticles. Such synthesis technique of noble nanoparticles for applications in catalysis, electronics, environmental and biotechnology is an area of constant interest [10-11]. It is very clear that nanoparticles can be synthesized and stabilized by using chemical methods such as chemical reduction [12-13], electrochemical techniques [14], photochemical reactions [15] and now a days via green chemistry route [16]. With the voracious demand of nanoparticles, use of plant extarcts in the synthesis of nanoparticles is quite novel, which give an added advantage over the chemical and physical methods, as it is cost effective and environment friendly. Such processes can be easily scaled up avoids the usage of high pressure, energy, temperature and toxic chemicals. Recently researchers are using bacteria and fungi for the synthesis of nanoparticles [17-23]. It is further suggested that use of leaf extract [24-25] is cost effective as it does not require any special culture preparation and isolation techniques. But the major problems underlying in these processes is maintaining the monodisperse nature of the nanoparticles and also the rate of the synthesis process, which is quite slow.

In the present synthesis route, a new path for the synthesis of silver nanoparticles was developed by using Ocimum leaf (Indian name -Tulasi) extract and microwave irradiation. Tulasi is a very common plant which is ordinarily found in every Indian household. It has been reported to contain alkaloids, glycosides, tannins, saponins and aromatic compounds [26]. It is used in the treatment of headaches, coughs, diarrhoea, constipation, worts, worms and kidney malfunctions. Recent interest in Ocimum has resulted from its inhibitory activity against HIV-1 reverse transcriptase and platelets aggregation induced by collagen and ADP (adenosine 5-diphosphate) [25]. This synthesis work was 
carried out by K. Mallikarjuna et al. [25], however in the present synthesis, Ocimum leaf extract in combination with microwave irradiation was used which resulted in a significant change in the size, surface morphology and synthesis time of the nanoparticles.

\section{Materials and Method}

Chemicals. All the experiments were conducted at room temperature. Materials used for the synthesis of silver nanoparticles are $\mathrm{AR}$ grade silver nitrate $\left(\mathrm{AgNO}_{3}\right)$ purchased from Merck, India, fresh leaves of Osmium leaf are collected from M.A.N.I.T campus and deionized water. Microwave (IFM Model 30SC3) of fixed freq. $2450 \mathrm{MHz}$, power source $1400 \mathrm{~W}$ and power consumption $900 \mathrm{~W}$ was used for heating of leaf extract to suppress the enzymes present in the leaf and for the synthesis of AgNP.

Preparation of Ocimum Leaf Extract. Fresh leaves of Ocimum were collected from campus of MANIT BHOPAL, India. The leaf extract used was prepared by taking $10 \mathrm{gm}$ of finely cut leaf into appropriate size and washed several times with deionised water. In a $250 \mathrm{ml}$ wide neck borosil beaker containing $200 \mathrm{ml}$ of distilled water, these washed leaves were added. The contents were mixed well and then the mixture was kept in microwave oven for $180 \mathrm{sec}$ to suppress the enzymes present in the solution. Further, the extract obtained was filtered in hot condition to remove fibrous impurities. The resultant clear extract was stored at $4{ }^{\circ} \mathrm{C}$ and used for synthesis process.

Synthesis of Colloidal Silver Nanoparticles. Aqueous solution of $10^{-3} \mathrm{M}$ of $50 \mathrm{ml}$ silver nitrate $\left(\mathrm{AgNO}_{3}\right)$ was prepared and used for the synthesis of silver nanoparticles. To this solution, $10 \mathrm{ml}$ leaf extract was added and then the solution mixture was exposed to microwave irradiation of fixed freq. 
$2450 \mathrm{MHz}$, until the color of the colloidal solution became yellowish brown. The color change of the solution indicates formation of silver nano particles by reduction of $\mathrm{Ag}^{+}$. Periodically, aliquots of the reaction solution were removed after time interval of $0,10,30,50,70,90,150$ and $300 \mathrm{sec}$ and subjected to UV-Vis spectroscopy measurements.

Methods. The formation of the AgNP was monitored using UV-Vis spectroscopy by employing a Systronics Double beam UV-Vis spectrophotometer operated on 300-800 nm wavelength. UV-Vis analysis of the reaction mixture was observed for a period of $300 \mathrm{~s}$. Distilled water was used as a solvent for UV study. For the crystalline studies, films of colloidal Ag nanoparticles formed on $\mathrm{Si}$ substrates by drop coating were used for X-ray diffraction (XRD) study. The formation was checked by X-ray diffraction (XRD) spectrum (Rigaku MiniflexII) of characteristic Co $30 \mathrm{Kv} / 15 \mathrm{miliA}$ radiation $\left(\lambda=1.54 \mathrm{~A}^{\circ}\right)$ in the range of $20^{\circ}$ to $90^{\circ}$ at a scan rate of $5^{\circ} / \mathrm{min}$. The transmission electron microscopy (TEM) image of the sample was obtained using JEOL Model JEM-1400 instrument. Sample for TEM analysis were prepared by coating of aqueous AgNP drops on carbon-coated copper grids, kept for $5 \mathrm{~min}$; the extra solution was removed using blotting paper. The film of the TEM grid is exposed to IR light for 30 min for drying.

\section{Results and Discussion}

The elemental analysis of as-synthesised silver nanoparticles was done using XRD (Fig. 1). XRD results confirm the crystallinity and FCC structure of the nanoparticles. XRD peaks appear at $38.11^{\circ}$, $44.20^{\circ}, 64.52^{\circ}$ and $77.51^{\circ}$ due to reflections from (111), (200), (220) and (311) planes respectively. All the four peaks obtained in the XRD graph matched well with standard JCPDS: 03-0931 of silver, which confirmed the formation of silver. However, the diffraction peaks were broad as compared to bulk, indicating that the crystal size was small. 


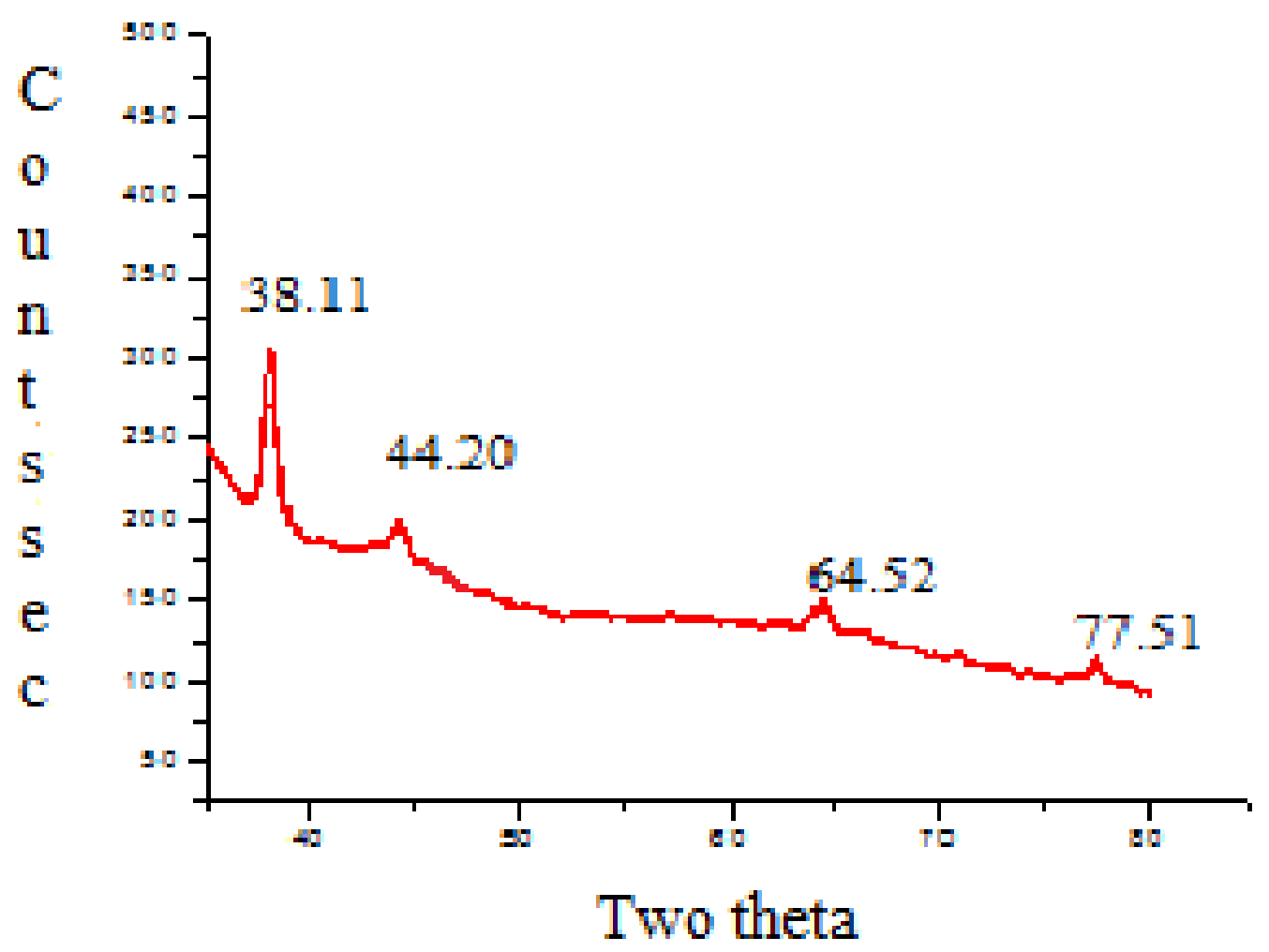

Fig. 1. Typical X-ray diffraction (XRD) pattern of the synthesised silver nanoparticles formed by using microwave irradiation time as $300 \mathrm{sec}$.

The next characteristic study which was done to study the morphology and crystal structure of the synthesized nanoparticles was TEM. The results of TEM image of the sample prepared by $300 \mathrm{sec}$ microwave irradiation are shown in Fig. 2. By careful observation of the TEM images it seems to be very clear that the nanoparticles are surrounded by a very faint layer of organic compounds. These organic compounds are those compounds which are responsible for reduction of $\mathrm{Ag}^{+}$ion as well for stability of nanoparticles [25]. The groups present in Ocimum leaf extract are proteins and metabolites such as terpenoids having functional groups of amines, alcohols, ketones, aldehydes and carboxylic acids [25]. The FTIR studies by K. Mallikarjuna et al. [25] found that the carbonyl group from the amino acid residues and proteins has the stronger ability to bind metals, indicating that the proteins could possibly be responsible for capping of the synthesised nanoparticles and also prevent them from agglomeration. This suggests that the biological molecules could possibly perform dual 
functions of formation and stabilization of silver nanoparticles in the aqueous medium [27]. So by this study a very good agreement can be made with the literature, about the bio-functionalization i.e., the nature of capping conferring the stability to the silver nano particles.
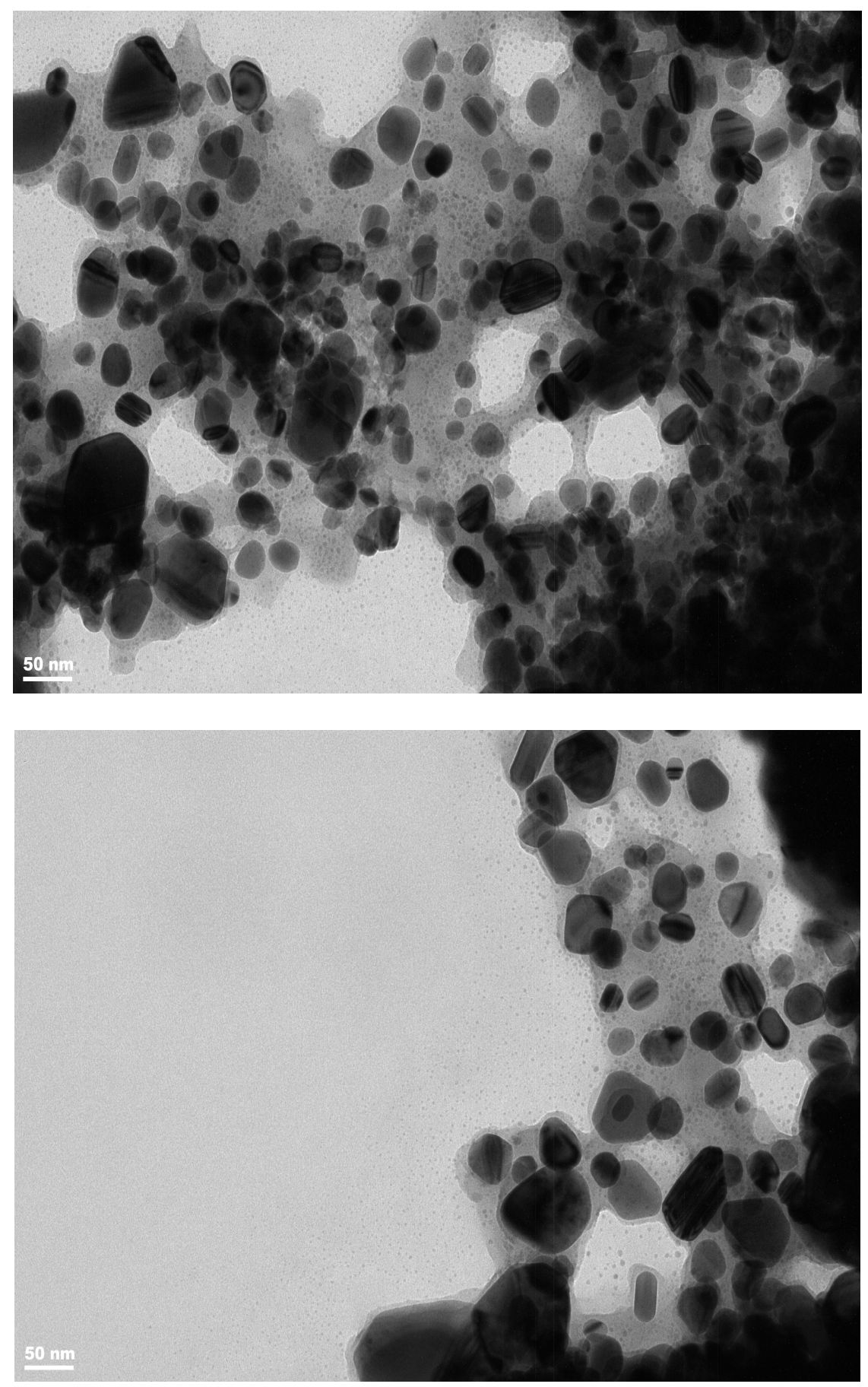

Fig. 2. TEM images of synthesised silver nanoparticles formed by using time of microwave irradiation as $300 \mathrm{sec}$. 
The results of TEM indicated that the average particle size was in the range of 5-50 nm.TEM images also show variation in the shape of the nanoparticles, which is due to the bio synthesis route adopted by the author [28]. By this route, mostly the synthesised nanoparticles are in spherical and oval shape. The reason for this type of shape is due to the fact that when a particle is formed, its initial state tries to acquire a shape that corresponds to minimum potential energy. The spherical and oval shapes correspond to state of minimum potential energy. By careful observation of the images we can say that the particles are surrounded by a faint layer of motives responsible for the internal binding of the nano particles. Further studies can be done on the effect on shape and size of the nanoparticles by increasing the concentration of leaf extract in presence of microwave irradiation.

The main advantage of microwave irradiation method is that the initial heating process is rapid, so uniform heat can be transferred to the solution and one more reason is that the microwave induces the generation of localized high temperatures of reaction sites, which increases the reaction rates and make synthesis process rapid [29-30]. Microwave offers a rapid and volumetric heating of solvents, reagents, and intermediates that provides uniform nucleation and growth conditions for nanomaterial synthesis [31-35]. The next characterization of the nanoparticles was done using UV-Vis spectroscopy. It is very important in respect to silver nanoparticles because UV-Vis is the most common method to investigate surface plasmon resonance. The distinctive colors of colloidal silver are due to a phenomenon known as plasmon absorbance. Incident light creates oscillations in conduction electrons on the surface of the nanoparticles and electromagnetic radiation is absorbed. As the leaf extract was added to the aqueous solution of $\mathrm{AgNO}_{3}$ solution, it started to change the color from watery to yellow due to reduction of silver ion which indicates formation of silver nanoparticles. The wavelength of the plasmon absorption maximum in a given solvent can be used to indicate particle size. The intensity of absorption band increases with increasing time period as well as concentration of capping agent and color changes were observed from colorless to yellowish brown [27]. The UV -Vis spectra of synthesised nanoparticles is shown in Fig.3. The surface plasmon peaks obtained confirm the reduction of $\mathrm{Ag}^{+}$to silver nanoparticles by Ocimum leaf extract 
in the presence of microwave irradiation. From Fig. 3 it is evident that the reaction intensity is a function of exposed time i.e. as the time increases the intensity increases. The SPR peak is obtained at $485 \mathrm{~nm}$. On close observation of the UV-Vis spectra of the samples having different expose time, we can easily see a blue shift in the peaks of growing intensity. This is probably due to the slight change in the size and shape of the synthesised nanoparticles [36]. The effect of time on absorption intensity is clear from Fig.4. We can also conclude from the graph that $90 \%$ of the reaction is completed in 90 s.

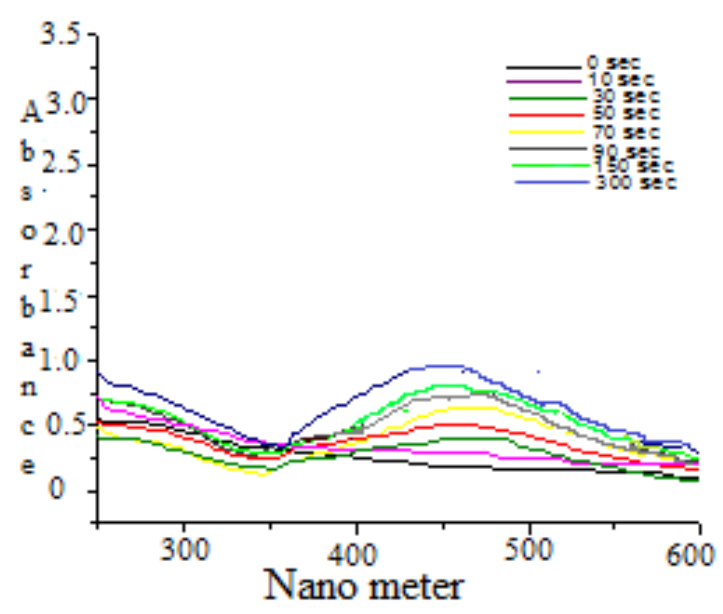

Fig. 3. UV-Vis spectra of synthesised Ag nano particles at different microwave irradiation time.

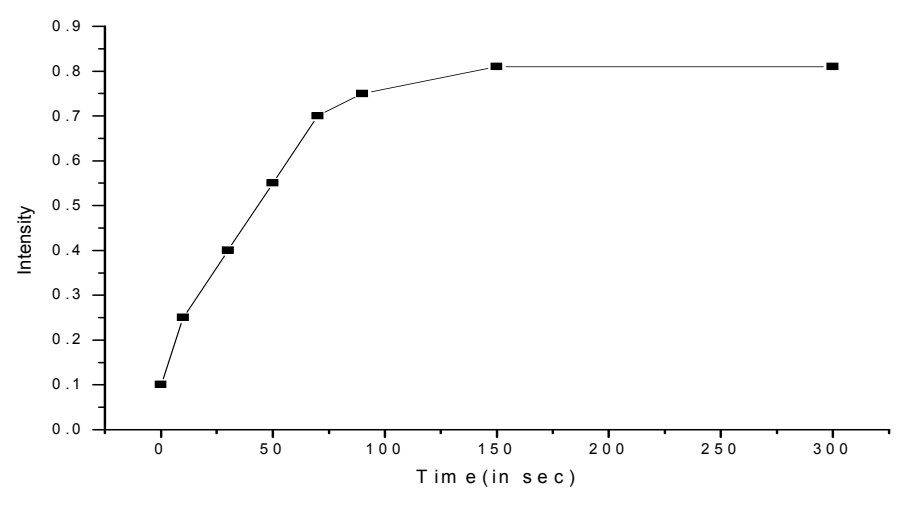

Fig. 4. Intensity graph of reaction mixture containing aqueous $\mathrm{AgNO}_{3}$ solution treated with microwave exposed leaf extract versus time. 


\section{Conclusions}

The present synthesis route is an eco-friendly, efficient, simple and fast process for synthesis of silver nanoparticles. To the best of our knowledge, using microwave irradiation with additions with Ocimum leaf extract, is the most rapid and fastest process among all the process reported in theliterature and also this is the main feature which makes the process so important and unique. The synthesised nano particles are spherical and oval in shape ranging from 5-50 nm in size. As reported in the literature this particular shape and size particles are often used in antimicrobial/anti cancer studies and to increase the solar cell efficiency [36,37]. Also because of using green route instead of using hazardous chemicals definitely the synthesised silver nanoparticles can be environment friendly. Though more study is required to understand the mechanism of this nano particle formation, size and shape contraol etc., but this a prelimary report towards a green synthetic approach.

\section{Acknowledgement}

The author acknowledges Director, MANIT Bhopal, for providing support and facilities to carry research work smoothly in the institute, AMPRI Bhopal for XRD Bhopal, HSADL for TEM facility.

\section{References}

[1] W. Jahn, Chemical aspects of the use of gold clusters in structural biology. J. Struct. Biol., 127 (1999) 106-112.

[2] H.S. Naiwa Ed. Hand Book of Non-structural Materials and Nanotechnology Academic, Press New York 1-5 (2000).

[3] C.J. Murphy, Sustainability as a Design Criterion in Nanoparticle Synthesis and Applications, J. Mater Chem.18 (2008) 2173-2176. 
[4] S. Schultz, D.R. Smith, J.J. Mock, D.A. Schultz, Single-target molecule detection with nonbleaching multicolor optical immunolabels, PNAS 97(3) (2000) 996-1001.

[5] M. Rai, A. Yadav, A. Gade, Silver nanoparticles: A novel antimicrobial agent, Biotechnology Advances 27 (2009) 76-83.

[6] J.L. Elechiguerra, J.L. Burt, J.R. Morones, A. Camacho-Bragado, X. Gao, H.H. Lara, M.J. Yacaman, Interaction of silver nanoparticles with HIV-1, J. Nanobiotechnol. 3(6) (2005). (doi:10.1186/1477-3155-3-6).

[7] R.M. Crooks, B.I. Lemon III, L. Sun, L.K. Yeung, M. Zhao, Dendrimer-Encapsulated Metals and Semiconductors: Synthesis, Characterization, and Applications, Top. Curr. Chem. 212 (2001) 82-135.

[8] D.I. Gittins, D. Bethell, R.J. Nichols, D.J. Schiffrin, Diode like electron transfer across nanostructured films containing redox ligands, J. Mater. Chem. 10 (2000) 79-83.

[9] I. Hussain, M. Brust, A.J. Papworth, A.I. Cooper, Preparation of acrylate-stabilized gold and silver hydrosols and gold-polymer composite films, Langmuir 19(11) (2003) 4831-4835.

[10] V.K. Sharma, R.A. Yngard, L. Yekaterina, Silver nanoparticles: Green synthesis and their antimicrobial activities, Colloid and Interface Science 145 (2009) 83-96.

[11] B. Krishna, D.V. Goia Dan, Silver nanoparticles for printable electronic biological applications, J. Mater. Res. 24(9) (2009) 2828-2836. 
[12] R.M. Tripathi, A. Saxena, N. Gupta, H. Kapoor, R.P. Singh, High antibacterial activity of silver nanoballs against E.COLI MTCC 1302, S. TYPHIMURIUM MTCC 1254, B. SUBTILIS MTCC 1133 and P. AERUGINOSA MTCC 2295, Digest Journal of Nanomaterials and Biostrucutres 5(2) (2010) 323-330.

[13] L. Rodriguez-Sanchez, M.C. Blanco, M.A. Lopez-Quintela, Electrochemical synthesis of silver nanoparticles, J. Phys. Chem. B 104 (2000) 9683-9688.

[14] A. Taleb, C. Petit, M.P. Pileni, Synthesis of highly monodisperse silver nano particles from AOT reverse micelles, a way to 2D and 3D self organization, Chem. Mater. 9 (1997) 950.

[15] N.A. Beguma, S. Mondalb, S. Basub, R.A. Laskara, D. Mandalb, Colloids Surf., B 71(1) (2009) 113-118.

[16] L. Maggy, S. Gordon, Bioaccumulation of gold by sulfate-reducing bacteria cultured in the presence of gold (I)-thiosulfate complex. Geochimica et Cosmochimic Acta 70(14) (2006) 3646-3661.

[17] B. Nair, T. Pradeep, Coalescence of Nano clusters and Formation of Submicron Crystallites Assisted by Lactobacillus Strains, Crystal Growth \& Design. 2(4) (2000) 293-298.

[18] S. He, Z. Guo, Y. Zhang, S. Zhang, J. Wan, N. Gu, A cost-effective preparation method of Ba-hexaaluminate nanoparticles for catalytic combustion of methane, Mater. Lett. 61 (2007) 3935-3938.

[19] J.D. Holmes, P.R. Smith, R. Evans-Gowing, D.J. Richardson, D.A. Russel, J.R.Sodeau, Energy dispersive X-ray analysis of the extracellular cadmium sulfide crystallites of Klebsiella aerogenes, Arch. Microbiol. 163(2) (1995) 143-147. 
[20] P. Mukherjee, S. Senapati, D. Mandal, A. Ahmad, M.I. Khan, R. Kumar, M. Sastry , Extracellular Synthesis of Gold Nanoparticles by the Fungus Fusarium Oxysporum, ChemBioChem 3 (2002) 461-463.

[21] A. Ahmad, P. Mukherjee, S. Senapati, M. Mandal, M.I. Khan, R. Kumar, M. Sastry, Extracellular Biosynthesis of Silver Nanoparticles using the Fungus Fusarium Oxysporum, Colloids Surf. B 28 (2003) 313-318.

[22] C.B. Kuber, S.F. D'Souza, Extracellular biosynthesis of silver nanoparticles using the fungus Aspergillus fumigates, Colloids Surf. B 47 (2006) 160-164.

[23] S.S. Shiv, R. Akhilesh, A. Absar, M. Sastry, Rapid synthesis of Au, Ag and bimetallic Au core-Ag shell nanoparticles using Neem (Azadirachta indica) leaf broth. J. Colloid Interface Sci. 275 (2004) 496-602.

[24] J.L. Gardea-Torresdey, J.G. Parsons, E. Gomez, J. Peralta-Videa, H.E. Troiani, P. Santiago, M.J. Yacaman, Formation and Growth of Au Nanoparticles inside Live Alfalfa Plants, Nano Letters 2 (2002) 397-401.

[25] K. Mallikarjuna, G. Narasimha, G.R. Dillip, B. Praveen, B. Shreedhar, C.S. Lakshmi, B.V.S. Reddy, B.D.P. Raju, Green Synthesis fo silver nanoparticles using ocimum leaf extarct and thier characterization, Digest Journal of Nanomaterials and Biostructures, 6 (1) (2011) p. 181-186.

[26] K. Yamasaki, M. Nakano, T. Kawahata, H. Mori, T. Otake, N. Ueba, I. Oishi, R. Inami, M.Yamane, M. Nakamura, H. Murata, T. Nakanishi, Anti-HIV-1 activity of herbs in Labiates, Biol. Pharm. Bull. 21(8) (1998) 829-833. 
[27] N. Ahmad, S. Sharma, V.N. Singh, S.F. Shamsi, A. Fatma, B.R. Mehta, Biosynthesis of Silver Nanoparticles from Desmodium triflorum, Novel Approach Towards Weed Utilization, Biotechnology Research International. 454090 (2011) 8.

[28] S. Navaladian, B. Viswanathan, T.K. Varadarajan, R.P. Viswanath, Microwave-assisted rapid Synthesis of anisotropic Ag nanoparticles by solid state transformation, Nanotechnology. 19 (4) (2008) 045603.

[29] S. Navaladian, B. Viswanathan, T.K. Varadarajan, R.P. Viswanath, Thermal decomposition as route for silver nano particles, Nanoscale Research Letters. 2 (2008) 44-48.

[30] W.J. Sommer, M. Weck, Facile Functionalization of Gold Nanoparticles via MicrowaveAssisted 1,3 Dipolar Cycloaddition, Langmuir 23 (2007) 11991-11995.

[31] J.A. Gerbec, D. Magana, A. Washington, G.F. Strouse, Microwave Enhanced Reaction Rates for nano particles synthesis, J. Am. Chem. Soc. 27 (2005) 15791-15800.

[32] V. Polshettiwar, M.N. Nadagouda and R. S. Varma, Chem. Commun, (2008), 6318.

[33] B. Baruwati, M. N. Nadagouda and R. S. Varma, Bulk Synthesis of Monodisperse Ferrite Nanoparticles at Water-Organic Interface under Conventional and Microwave Hydrothermal treatment and their surface functionalization, J. Phys. Chem. C 112 (2008) 18399-18404.

[34] M. N. Nadagouda and R. S. Varma, Microwave-Assisted Shape-Controlled Bulk Synthesis of Ag and Fe Nanorods in Poly(ethylene glycol) Solutions, Cryst. Growth Des. 8(1) (2008) 291-295. 
[35] Stephan L, Mostafa A, El-Sayed J, Size and temperature dependence of the plasmon Absorption of colloidal gold nanoparticles, Phys. Chem. B 103 (1999) 4212-4217.

[36] T.K. Jeorger, R. Jeorger, E. Olsson, C. Granqvist Bacteria as workers in the living factory:metal-accumulating bacteria and their potential for materials science, Biotechnol. 19(1) (2001) 15-20.

[37] Q. Wu, H. Cao, Q. Luan, J. Zhang, Z Trends, Wang, J.H. Warner, A.A.R.Watt, Biomolecule-assisted synthesis of water-soluble silver nanoparticles and their biomedical applications, Inorg. Chem. 47(13) (2008) 5882-5888. 